\title{
Forecast of deformations of the land surface from the separate clearing development, displacement and deformation in the main sections of the trough
}

\author{
Tamara Makeeva ${ }^{1} *$ and Vitaly Trofimov ${ }^{2}$ \\ ${ }^{1}$ Moscow State University of Civil Engineering, Yaroslavskoe shosse, 26, Moscow, 129337, Russia \\ ${ }^{2}$ Russian Academy of Sciences, Institute of problems of complex development of bowels, 111020, 4 , \\ Kryukovsky tupik, Moscow, Russia
}

\begin{abstract}
Two approaches for the description of parameters of the trough of the land surface subsidence in the main sections (its forms, inclinations and curvature) are considered in comparison with each other. One of them, (the method of standard curves) makes up the basis for "Rules of the structures protection...", the document defining the approach to the assessment of deformation of the day surface during design of various facilities, located on it. Besides, the approach, developed in some other works by the authors, based on the approximation of the form of the trough subsidence with the functions of the probabilities integrals type with three defining parameters, is considered. The attempt to express the standard curves as the mention functions is undertaken. It is proved that it is possible to define the values of three corresponding parameters of the approximating functions. Thereby the tabular dependences are transformed to the analytical relations, correct for all the range of the parameters change, i.e. not just for those, recorded in the tables.
\end{abstract}

\section{Introduction}

The development of coal layers is followed by the change of stress of rocks and their displacement $[1,2,3]$, perceptible in the formation of a zone of displacement in the massif and on the land surface. The displacement zone on a surface, appearing from the influence of separate clearing development, is called a displacement trough.

Separate points in the displacement trough move by different sizes in the considered timepoint, therefore vertical deformations (inclinations, curvature, twisting) and horizontal deformations (stretching, compression, shifts) as well as washout and the concentrated deformations (cracks and ledges in the vertical plane and shifts in the horizontal plane) arise.

Displacements and deformations of rocks and the land surface can cause damages to the facilities, the increase in water permeability and gas permeability of the breeds over the

Corresponding autour: makeeva13new@yandex.ru 
developed space, change of the hydrogeological mode of surface and ground waters, activization of landslide processes.

The sizes of the zone of influence of underground minings, sizes and nature of deformations, the rapidity of the deformations growth and the duration of the process of displacement of rocks and land surface depend on the following major factors:

a) capacity, hade and the depth of the developed layers;

b) size of the clearing developments, the sequence of passing and mutual position of borders of the clearing developments in one layer and in the suite of layers;

c) structural features of the rocks massif (the structure of the containing breeds, existence of explosive and the plicative breakings, variability of breeds bedding and physical-and-mechanical properties of breeds;

d) the way of the mountain pressure control;

e) rapidity of the face movement and the rapidity of the development of works crosswise the stretch.

We consider the features of calculation of the land surface subsidence parameters within the method of standard curves, which became the basis for "Rules of protection of structures and natural objects from the adverse effect of underground mountain minings in the coal fields" [4], along with the semi-analytical method [5, 6], which uses the functions of the Gauss probabilities integral type for the approximation of the subsidence trough form. It should be noted that the regularities, put in its basis, are the synthesis of numerous natural observations in the mines of various coal basins and fields.

\section{Methods and algorithms}

According to [4] the maximum subsidence of the land surface is determined with the help of the formula:

$$
\eta_{m}=q_{0} m N \cos \alpha
$$

where $\mathrm{q}_{0}$ is the relative maximum subsidence; $\mathrm{m}$ is the taken-out layer thickness; the effective thickness is used during the work with a laying; $\alpha$ is a layer hade; $\mathrm{N}\left(\mathrm{N}_{1}\right.$ and $\left.\mathrm{N}_{2}\right)$ are the coefficients, connected with the relative depth of the development placement, i.e. with the relation of the development length (along extension and crosswise extension) to its depth. Size $\mathrm{q}_{0}$ is connected to the extent of loosening of breeds of the roof rocks in case of their failure into the developed space and has to be assessed separately for each field or basin.

The land surface subsidence in the points of the main sections of the displacement trough is determined according to the formula:

$$
\eta_{x y}=\eta_{m} S(z)
$$

where $S_{(z)}$ is the function of the standard curve of subsidence, determined by the tables [1] for the respective basins (fields), depending on $\mathrm{N}_{1}$ coefficients for the points of the main section crosswise the layers stretch and $\mathrm{N}_{2}$ for the points of the main section along the layers stretch; $z_{x}=x / L_{3}$ for the points, located in the main section along the layers stretch; $\mathrm{zy}_{1}=\mathrm{y}_{1} / \mathrm{L}_{1}$ for the points, located in the main section crosswise the layers stretch in the semitrough along the layer fall; $\mathrm{zy}_{2}=\mathrm{y}_{2} / \mathrm{L}_{2}$ for the points, located in the main section crosswise the layers stretch in the semi-trough along the layer rise; $x, y_{1}, y_{2}$ are the distances from the point of the maximum subsidence (the origin of coordinates) to the considered point, respectively in semi-troughs along the layer stretch, fall and rise; $\mathrm{L}_{1}, \mathrm{~L}_{2}, \mathrm{~L}_{3}$ are the lengths of the corresponding semi-troughs. Further such a table for coal fields of East Donbass is provided for an example. 
Table 1. Values of the functions $S_{(\mathrm{z})}, S_{(\mathrm{z})}^{\prime}, S^{\prime \prime}{ }_{(\mathrm{z})}$ for East Donbass

\begin{tabular}{|c|c|c|c|c|c|c|c|c|c|c|c|c|}
\hline \multirow{2}{*}{$z$} & \multicolumn{3}{|c|}{$N=1$} & \multicolumn{3}{|c|}{$N=0.9$} & \multicolumn{3}{|c|}{$N=0.8$} & \multicolumn{3}{|c|}{$N \leq 0.7$} \\
\hline & $\mathbf{S}(\mathbf{z})$ & $\mathbf{S}^{\prime}(\mathbf{z})$ & $\mathbf{S}^{\prime \prime}(\mathbf{z})$ & $\mathbf{S}(\mathbf{z})$ & $\mathbf{S}^{\prime}(\mathbf{z})$ & $\mathbf{S}^{\prime \prime}(\mathbf{z})$ & $\mathbf{S}(\mathbf{z})$ & $\mathbf{S}^{\prime}(\mathbf{z})$ & $\mathbf{S}^{\prime /}(\mathbf{z})$ & $\mathbf{S}(\mathbf{z})$ & $\mathbf{S}^{\prime}(\mathbf{z})$ & $\mathbf{S}^{\prime \prime}(\mathbf{z})$ \\
\hline 0 & 1.00 & 0.0 & 0.0 & 1.00 & 0.0 & -4.3 & 1.00 & 0.0 & -7.4 & 1.00 & 0.0 & -9.4 \\
\hline 0.1 & 0.99 & 0.19 & -2.1 & 0.98 & 0.47 & -5.0 & 0.97 & 0.73 & -7.0 & 0.96 & 0.91 & -8.2 \\
\hline 0.2 & 0.95 & 0.56 & -5.1 & 0.90 & 1.02 & \begin{tabular}{|c|}
-6.1 \\
\end{tabular} & 0.85 & 1.36 & -5.6 & 0.83 & 1.59 & $\begin{array}{l}-5.2 \\
\end{array}$ \\
\hline 0.3 & 0.86 & 1.20 & $\begin{array}{l}-7.3 \\
\end{array}$ & 0.77 & 1.61 & $\begin{array}{l}-5.3 \\
\end{array}$ & 0.69 & 1.83 & -3.0 & 0.65 & 1.90 & $\begin{array}{l}-1.8 \\
\end{array}$ \\
\hline 0.4 & 0.71 & 1.89 & -5.7 & 0.58 & 1.98 & -1.8 & 0.48 & 1.91 & 0.7 & 0.46 & 1.85 & 2.3 \\
\hline 0.5 & 0.50 & 2.20 & 0.0 & 0.39 & 1.92 & 2.9 & 0.31 & 1.67 & 3.9 & 0.29 & 1.49 & 4.3 \\
\hline 0.6 & 0.29 & 1.89 & 5.7 & 0.22 & 1.46 & 5.7 & 0.17 & 1.20 & 5.1 & 0.16 & 1.04 & 4.6 \\
\hline 0.7 & 0.14 & 1.20 & 7.3 & 0.10 & 0.87 & 5.6 & 0.08 & 0.71 & 4.4 & 0.08 & 0.62 & 3.7 \\
\hline 0.8 & 0.05 & 0.56 & 5.1 & 0.04 & 0.42 & 3.7 & 0.03 & 0.35 & 2.8 & 0.03 & 0.32 & 2.3 \\
\hline 0.9 & 0.01 & 0.19 & 2.1 & 0.01 & 0.15 & 1.5 & 0.01 & 0.13 & 1.2 & 0.01 & 0.12 & 1.1 \\
\hline 1.0 & 0.0 & 0.0 & 0.0 & 0.0 & 0.0 & 0.0 & 0.0 & 0.0 & 0.0 & 0.0 & 0.0 & 0.0 \\
\hline
\end{tabular}

The $S_{(z)}^{\prime}$ and $S_{(z)}^{\prime \prime}$ functions are used for the assess of slopes of $i_{x}$ and curvature of $K_{x}$ of the trough surface in its main sections.

$$
i_{x}=\frac{\eta_{m}}{L_{3}} S^{\prime}\left(z_{x}\right), K_{x}=\frac{\eta_{m}}{L_{3}^{2}} S^{/ /}\left(z_{x}\right) \text {. }
$$

Figure 1 presents the curve $\mathrm{S}(\mathrm{z})$, drawn by the means of interpolation of data from the provided table for various $\mathrm{N}$ values, which are also presented. The points, reflecting the data from the table are put to the curve for $\mathrm{N}=1$.

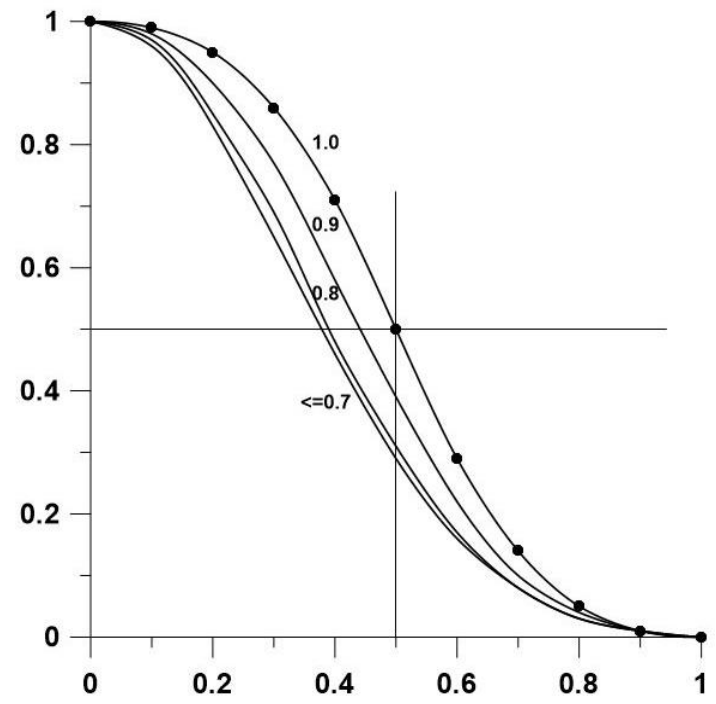

Fig. 1 Curves for S(z), drawn according to the Table 1

It should be noted that parameter $\mathrm{N}$ is determined by the ratio of lava length and the depth of clearing development placement. This dependence is presented in Figure 2. 


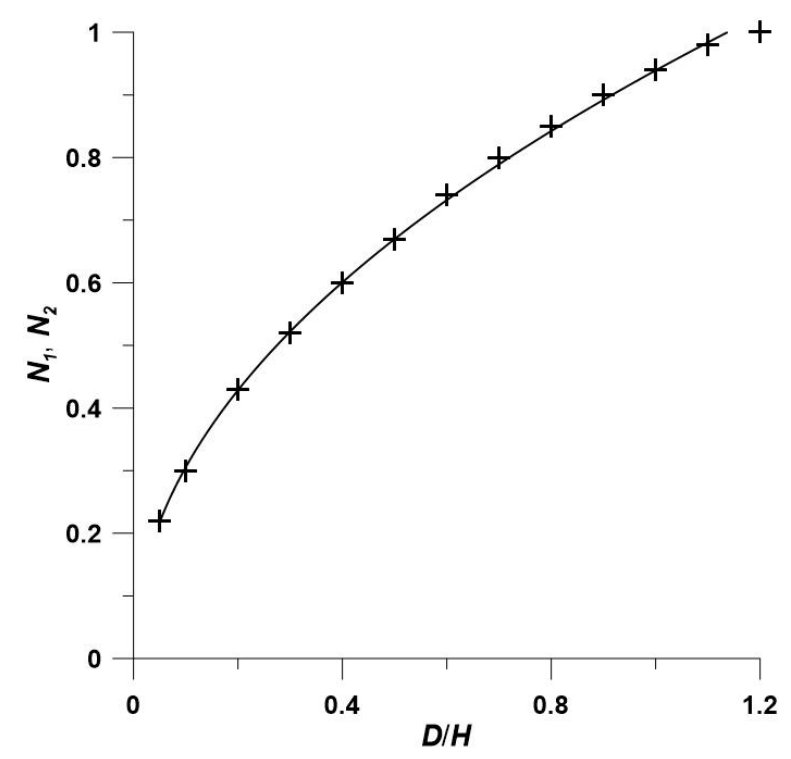

Fig. 2. Dependence of the parameters N1, N2 on the dimensionless of the development length

The corresponding analytical dependence is the following:

$$
N=0.939 *\left(\frac{D_{p}}{H}\right)^{0.487}
$$

Parameter $\mathrm{N}$ reflects the fact, that the closer development is located to the surface, the more is the sedimentation of the land surface according to (1) with other things being equal. At the same time the characteristic form of the trough in the main sections also changes. For the developments of rather shallow underlay, i.e. if $\mathrm{D} / \mathrm{H}>1.2$ it is possible to consider $\mathrm{N}=1$. In this case according to Figure 1 the symmetry of the lower and upper parts of the standard curve takes place. At the same time the symmetry point is $(0.5,0.5)$ and it is the point of discontinuity. The increase in the depth results in the curve asymmetry, though in insignificant degree.

Thus, the described approach fully solves the issue of prediction of the parameters of the land surface subsidence for the fixed values of determining parameters. However, the approximation of the provided tabular data is necessary for their intermediate values; that can be inconvenient for the practical application.

\section{Results}

The approximation of standard curves was carried out by the functions of the probabilities integral type.

Earlier [6-11] the approximation of the form of the curve of the land surface subsidence in the main section of the trough was used for the solution of a similar task. The following function was used:

$$
\eta(x)=-\frac{\eta_{0}}{2}\left[F\left(\frac{D_{p}+x}{C_{p} H}\right)+F\left(\frac{D_{p}-x}{C_{p} H}\right)\right]
$$

where

$$
F(t)=\sqrt{\frac{2}{\pi}} \int_{0}^{t} e^{\frac{z^{2}}{2}} d z
$$


It is possible to try to use these ratios for the approximation of the relevant data from Table 1 or the curves on Figure 1. As for Figure 3, the continuous lines show the curves of type (5) with the parameters $\eta_{0}, D_{p}, C_{p}$, chosen according to [10] for all $\mathrm{N}$ and, for comparison, the points show the data from the Table for $\mathrm{N}=0.7$ (as an example).

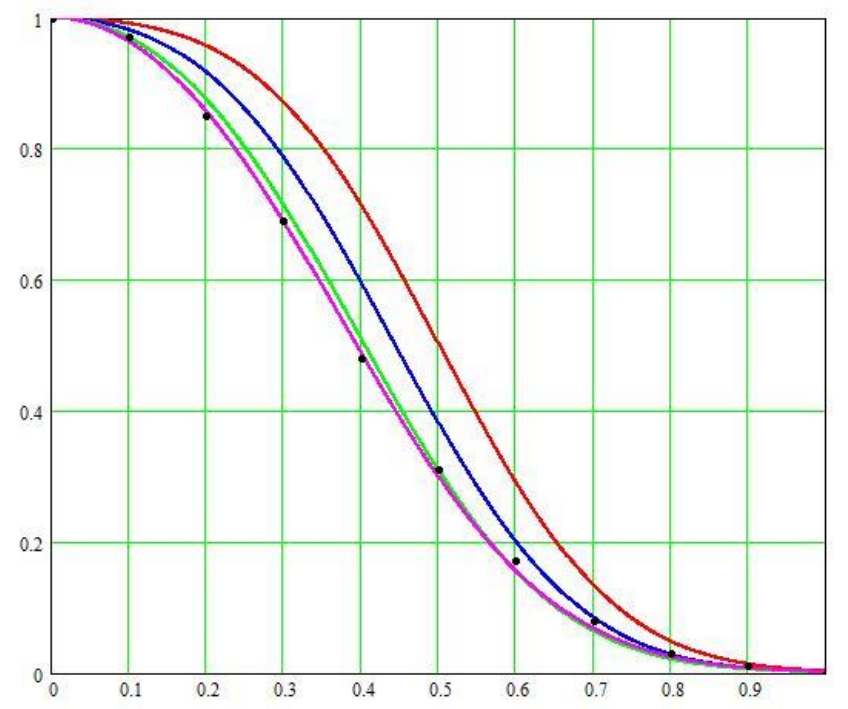

Fig. 3. Approximation of tabular data (Table 1) with the functions (5)

At the same time it was necessary to have $\mathrm{H}=1$ for providing the normalization of the received curve, which takes place for the curves in Figures 1, 3.

When using the interactive algorithm [10] the values of the parameter $\eta 0$, for all the curves (for all N) (Figure 1), as we would expect, appeared to be almost equal 1. As for the values of Dp and Cp, they are presented in Figure 4, where their dependence on D/H (1 and 2 respectively) is shown.

Analytically they are representable in the form of ratios:

$$
\begin{aligned}
& D_{p}=0.289+0.127 \frac{D}{H}+0.041\left(\frac{D}{H}\right)^{2}, \\
& C_{p}=0.291-0.187 \frac{D}{H}+0.079\left(\frac{D}{H}\right)^{2} .
\end{aligned}
$$

It should be noted that the tables of standard curves are made for rather narrow range of change of values of $\mathrm{N}$, i.e. $\mathrm{D} / \mathrm{H}$ (points represent the tabular values). The monotony and smoothness of the curves in Figure 4 allows to consider, that outside this range the values of the corresponding parameters can be received by extrapolation of the given curves. 


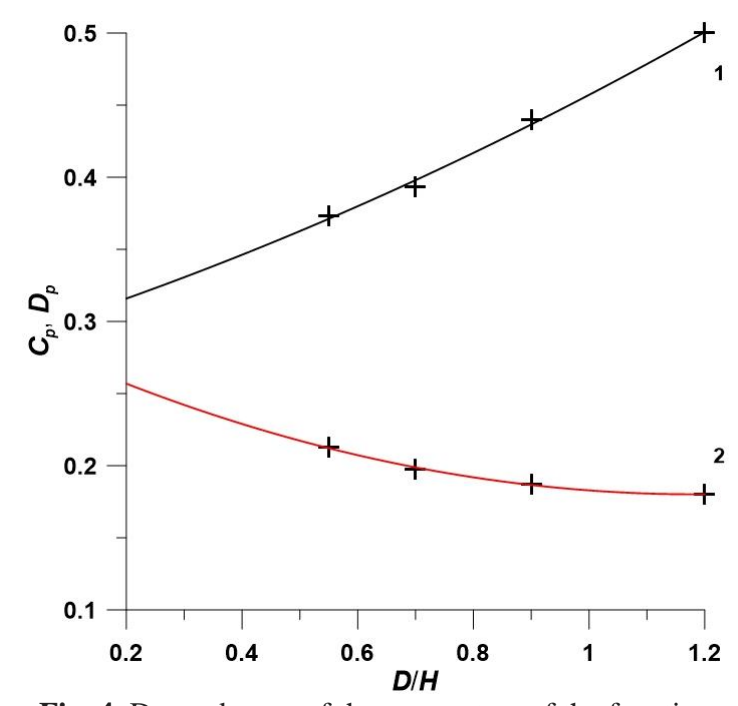

Fig. 4. Dependences of the parameters of the functions

(5) on the dimensionless extent of the development

The slope of the trough surface appears to be the differentiation of the expression (4) for the land surface subsidence. As a result we receive the following ratio:

$$
\eta^{\prime}(x)=-\frac{\eta_{0}}{2 C H} \sqrt{\frac{2}{\pi}}\left[e^{-\frac{1}{2}\left(\frac{D+x}{C H}\right)^{2}}-e^{-\frac{1}{2}\left(\frac{D-x}{C H}\right)^{2}}\right]
$$

Figure 5 represents the curves (9) for the values of parameters $\eta_{0}, D_{p}, C_{p}$, received earlier and corresponding to the values $\mathrm{N}-1,0.9,0.8,0.7$, or $\mathrm{D} / \mathrm{H}-1.2,0.9,0.8,0.7$. Here the points show the values of the $\mathrm{S} /$ ( $\mathrm{z}$ ) function from Table 1 for $\mathrm{N}=0.7$ (for comparison). It is possible to note that they coincide with the corresponding curve rather well.

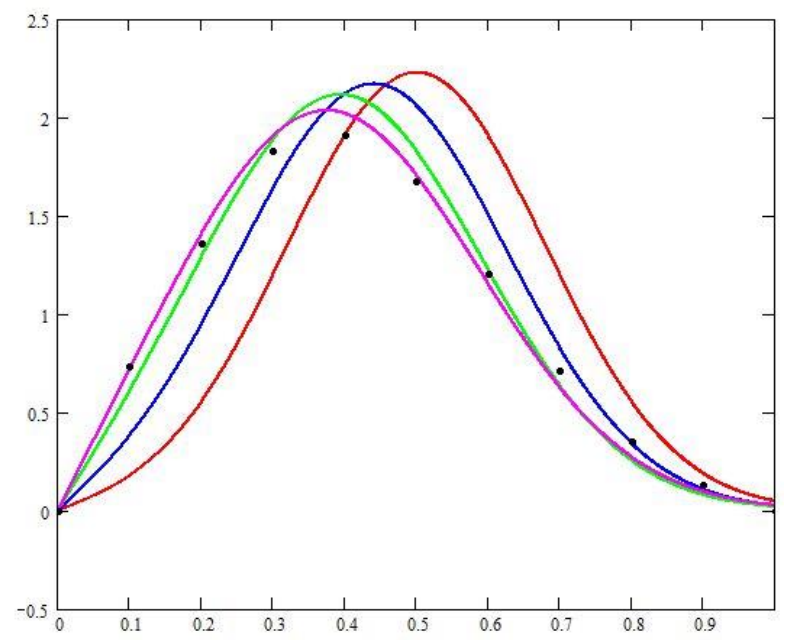

Fig. 5. Trough surface slope according to the approximation by the functions $(5,6)$

The received ratios (4)-(9) allow to estimate the parameters of the land surface deformation depending on its extent, rated on the depth of its the development, for each 
concrete field. It is necessary to use the aforesaid values of parameters $\eta_{0}, D_{p}, C_{p}$ for this purpose, for example for East Donbass (Figure 4).

\section{Conclusions}

We can conclude, that the carried out research proved the almost complete coincidence of the results, describing the parameters of deformation of the land surface both by the means of standard curves, and with the functions of the probabilities integral type. As the first approach synthesis of natural measurements "in situ" can be considered, which is not raising any doubts in the validity, can be considered, and the second approach should be also considered adequate thanks to the identity of results. Moreover, the second approach gives the opportunity to approximate the standard curves for various basins and fields. Thus, each such object can attribute values of three determining parameters $\eta_{0}, D_{p}, C_{p}$; that determining the nature of the land surface deformation depending on the rated extent of the developed space (lava).

The research is carried out with the financial support of the Russian Foundation for Basic Research No. 180500936.

\section{References}

1. S. Smiths, V. Trofimov, FTPRPI, 4, 1-22 (2007)

2. K. Trubetskoi, M. Lofis, S. Kuznetsov, V. Trofimov, Journal of mining science, Consultants Bureau, New York, 35-3, 209-215 (1999)

3. S. Kuznetsov, V. Trofimov, Geodynamics and tension of the subsoil of Earth (Novosibirsk, 2009)

4. Rules of protection of structures and natural objects from the adverse effect of underground mining operations on the coal fields (St-Peresburg, Interindustrial-andscientific center VNIMI, 1998)

5. G. Cratch, Movement of rocks and protection of underworked structures (Moscow, Nedra, 1978)

6. R. Muller, Influence of excavations on deformation of the land surface (Moscow, Ugletekhizdat, 1958)

7. S. Kuznetsov, V. Trofimov, Marksheyderiya of Kazakhstan: state and prospects, 119125 (2006)

8. S. Kuznetsov, V. Trofimov, Mountain journal of Kazakhstan, 7, (2006)

9. T. Makeeva, V. Trofimov, MATEC Web of Conferences, 117, 00107 (2017), DOI: https://doi.org/10.1051/matecconf/201711700170

10. T. Makeeva, V. Trofimov, IOP Conf. Series: Materials Science and Engineering, 365, 042025 (2018), doi:10.1088/1757-899X/365/4/042025.

11. T. Makeeva, V. Trofimov, MATEC Web Conf., 251, (2018) DOI:https://doi.org/10.1051/matecconf/201825102013 